\title{
Diagnostic simultané de champs de vitesse et de taille de particules
}

Les écoulements diphasiques à phase dispersée sont présents dans de nombreux procédés industriels tels que la filtration, la décantation, l'injection, la combustion... Ils sont caractérisés par la présence simultanée d'une phase continue, encore appelée phase porteuse (gaz), et d'une phase présente sous la forme d'inclusions (particules solides ou liquides), dite dispersée. Les mesures expérimentales de la taille, le spectre de répartition et la vitesse des particules sont fondamentales pour l'étude de ces écoulements ainsi que pour le développement des modèles de calcul. La maîtrise et l'optimisation de ces systèmes nécessitent des mesures expérimentales simultanées in situ, sans perturber l'écoulement, de la vitesse et de la taille des particules.

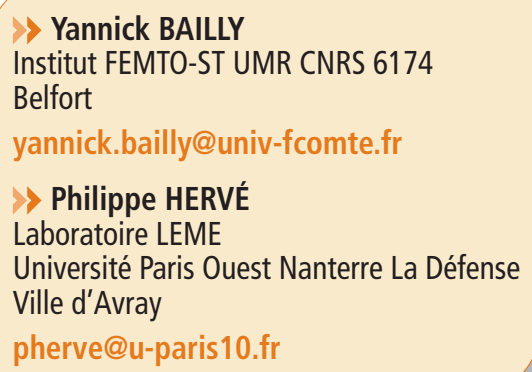

\section{Les techniques optiques utilisées}

Les principales techniques optiques permettant d'accéder à la taille de particules dans un écoulement sont regroupées dans le tableau 1. Elles peuvent être assemblées selon trois catégories en fonction du type de données traitées :

- la première s'applique à une analyse locale, c'est-à-dire qu'elle porte sur une petite région de l'écoulement. Les résultats obtenus sont interprétés par un modèle physique qui considère que le signal optique analysé résulte d'une particule individuelle. Ces méthodes nécessitent un arrangement géométrique rigoureux dans lequel les angles d'illumination et de collecte doivent être ajustés avec soin ;

- la seconde considère le signal lumineux émis par une assemblée de particules présentes dans une région de l'écoulement plus ou moins étendue. L'analyse n'est donc plus individuelle, mais globale; on parle de méthodes intégrales ;
- la dernière rassemble les techniques capables de fournir une information sur des particules individuelles dans une région de l'écoulement relativement vaste. On distingue alors les techniques dont I'éclairage est volumique, comme I'holographie en ligne (voir dans ce même numéro l'article de Corinne Fournier), et celles qui éclairent un plan de l'écoulement que l'on observe avec un dispositif d'imagerie. Dans les deux cas, on obtient les champs de taille de particules et de vitesse lorsque ces techniques sont couplées avec une technique de suivi de particules (PTV) ou de corrélation d'images (PIV ou flot optique).

Cet article mettra l'accent sur deux techniques de champs, complémentaires par leurs étendues de mesure respectives, basées sur de l'imagerie couplée à de la vélocimétrie par PIV ou PTV.

\section{Granulométrie par imagerie laser à défaut de mise au point (Interferometric Laser Imaging Droplet Sizing - ILIDS)}

Le principe de cette méthode est schématisé sur la figure 1. Elle consiste à éclairer un ensemble de gouttelettes avec un plan laser et recueillir leurs images en défocalisant légèrement le dispositif d'observation. On peut noter que cette méthode, décrite ici pour des gouttelettes, peut s'appliquer aussi à d'autres particules, pourvu qu'elles soient transparentes, notamment à des bulles.

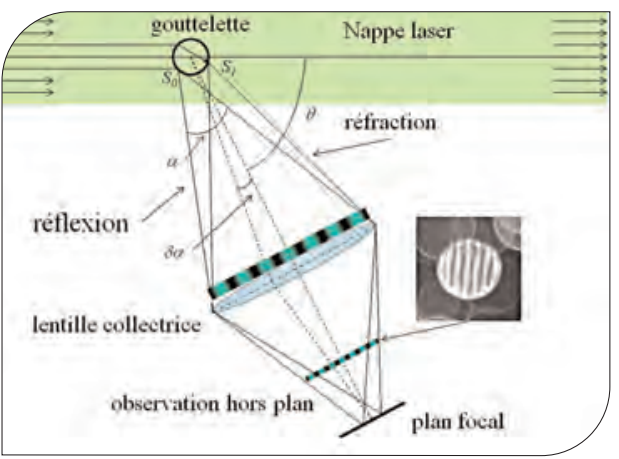

Figure 1. Principe de la méthode.

Pour chaque gouttelette, tout se passe alors comme si les deux sources ponctuelles $S_{0}$ et $S_{1}$, formées respectivement par les rayons réfléchis et réfractés, formaient un champ d'interférences dans le plan du CCD. Chaque gouttelette apparaît alors comme un disque rempli d'un réseau de franges. L'espace angulaire entre les franges dépend directement de la distance entre $S_{0}$ et $S_{1}$ et donc du diamètre de la particule [König et coll.]. II suffit alors de compter les franges présentes dans chaque disque pour déduire la taille de la gouttelette correspondante. Compte tenu du montage optique et notamment de l'angle de collection de la lumière, le diamètre des gouttelettes s'obtient avec la relation suivante :

$$
d=\frac{2 \lambda N}{\alpha}\left[\frac{n \sin (\theta / 2)}{\sqrt{n^{2}-2 n \cos (\theta / 2)+1}}+\cos (\theta / 2)\right]^{-1}
$$

Pour des bulles dans un liquide, la relation doit être modifiée comme suit :

$$
d=\frac{2 \lambda N}{\alpha}\left[n \cos (\theta / 2)-\frac{n \sin (\theta / 2)}{\sqrt{n^{2}-2 n \cos (\theta / 2)+1}}\right]_{\gg}^{-1}
$$


Tableau 1. Récapitulatif des techniques existantes [Onofri].

\begin{tabular}{|c|c|c|c|c|c|c|c|c|c|}
\hline & & 0,01 & 0,1 & 0,5 & 1 & 10 & 100 & 1000 & \\
\hline \multirow{3}{*}{ Particules individuelles } & PDPA (Phase Doppler Particle Analyser) & & & & & & & & \\
\hline & Arc-en-ciel & & & & & & & & \\
\hline & Rapport d'intensité & & & & & & & & \\
\hline \multirow{3}{*}{$\begin{array}{l}\text { Nuage de particules } \\
\text { (Méthodes intégrales) }\end{array}$} & PCS (Photon Correlation Spectroscopy) & & & & & & & & \\
\hline & Exctinction Spectrale & & & & & & & & \\
\hline & Diffraction Fraunhofer & & & & & & & & \\
\hline \multirow{5}{*}{ Techniques de champ } & Holographie (+ corrélation ou PTV) & & & & & & & & \\
\hline & LIF (Laser Induced Fluorecence) (+ PIV) & & & & & & & & \\
\hline & Ombroscopie (+ PTV) & & & & & & & & \\
\hline & Imagerie défocalisée (+ PTV) & & & & & & & & \\
\hline & Tomographie + rapport d'intensité (+ PIV) & & & & & & & & \\
\hline
\end{tabular}

L'analyse montre une faible sensibilité de l'indice de réfraction de la gouttelette et de l'angle d'observation $\theta$ sur la détermination de son diamètre. En particulier, la méthode fonctionne parfaitement bien pour $\theta=\pi / 2$, ce qui est très favorable pour faire une image du plan d'analyse. Par ailleurs (figure 2), la dynamique de la mesure est imposée par l'angle de collecte, c'est-à-dire l'ouverture numérique du système d'observation. En l'absence de défocalisation, on recueille simplement deux points lumineux appelés points de gloire, images de $S_{0}$ et $S_{1}$.

Cette méthode a été appliquée à l'étude d'un spray généré à partir d'un liquide proche de l'eau. La figure 3a montre le type de résultat que l'on obtient en exploitant une image enregistrée sur une caméra (CCD de 11 MPixel). En appliquant cette méthode couplée à une technique de vélocimétrie par éléments de trajectoires [Prenel \& Bailly], on peut montrer la correspondance entre la vitesse et la taille de bulles de $\mathrm{CO}_{2}$ dans un liquide (figure $3 b$ ).

Les limites de la méthode dépendent directement de la capacité du dispositif d'observation à compter les franges. Il est donc capital de limiter le nombre de particules dans l'image (ordre de grandeur : 300) en ajustant la taille de la zone d'analyse de façon à réduire les chevauchements. Lorsque la direction de l'écoulement est relativement uniforme, certains auteurs ajoutent un filtre spatial devant le système d'observation, de façon à obtenir une portion seulement de la figure d'interférences ; chaque particule apparaît alors comme une série de tirets lumineux

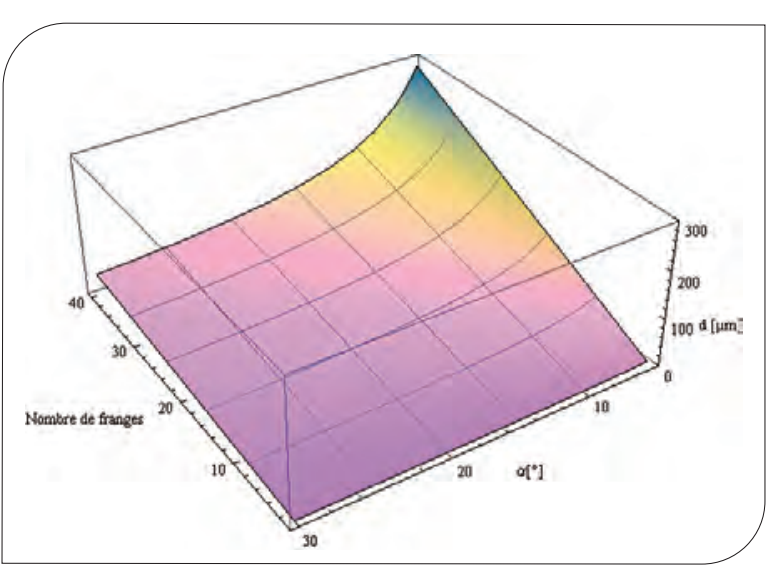

Figure 2. Diamètre mesuré en fonction de l'angle de collecte pour $\theta=\pi / 2$. correspondant aux franges d'interférences.

Notons que l'interprétation du nombre de franges se base sur l'hypothèse de particules transparentes de forme sphérique. Cette dernière condition est parfois difficile à satisfaire pour les grosses particules. Enfin, pour des tailles de particules inférieures à $10 \mu \mathrm{m}$, le nombre de franges devient trop faible pour que la mesure reste pertinente.

\section{Granulométrie tomographique par taux de polarisation}

Lorsque la taille des particules diminue, peu de techniques de champ restent opérantes pour analyser des portions d'écoulement de grande taille (50 mm typi-

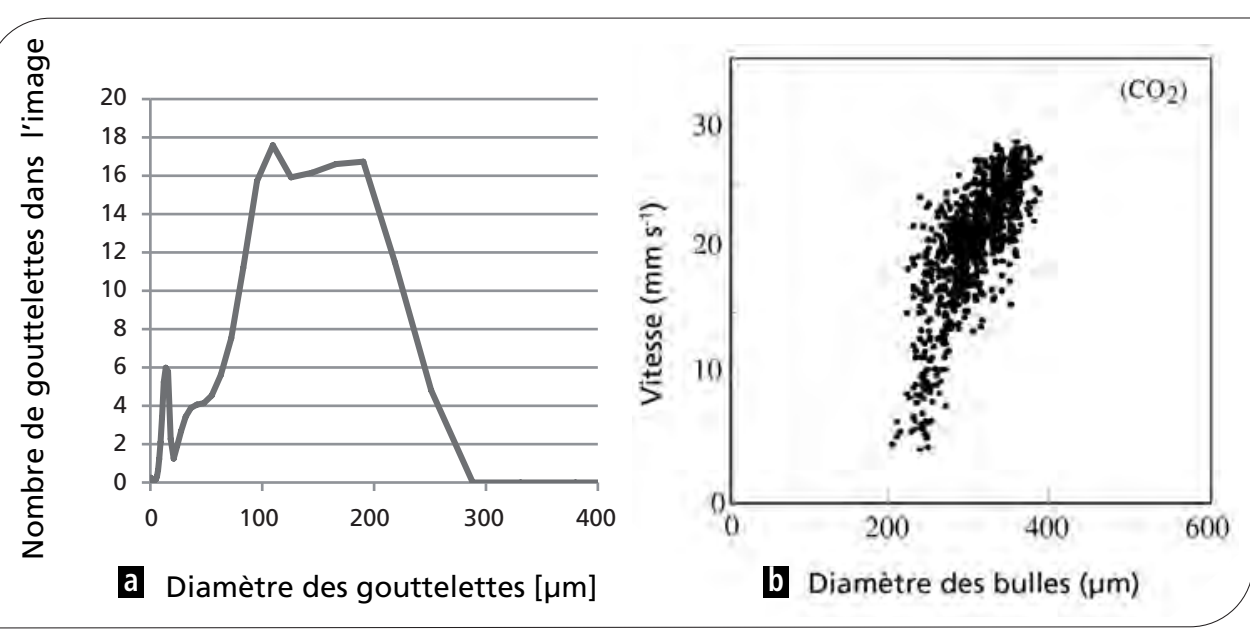

Figure 3. Application de la tomographie défocalisée à des gouttelettes ou des bulles : (a) analyse d'un spray, (b) analyse de bulles de $\mathrm{CO}_{2}$. 


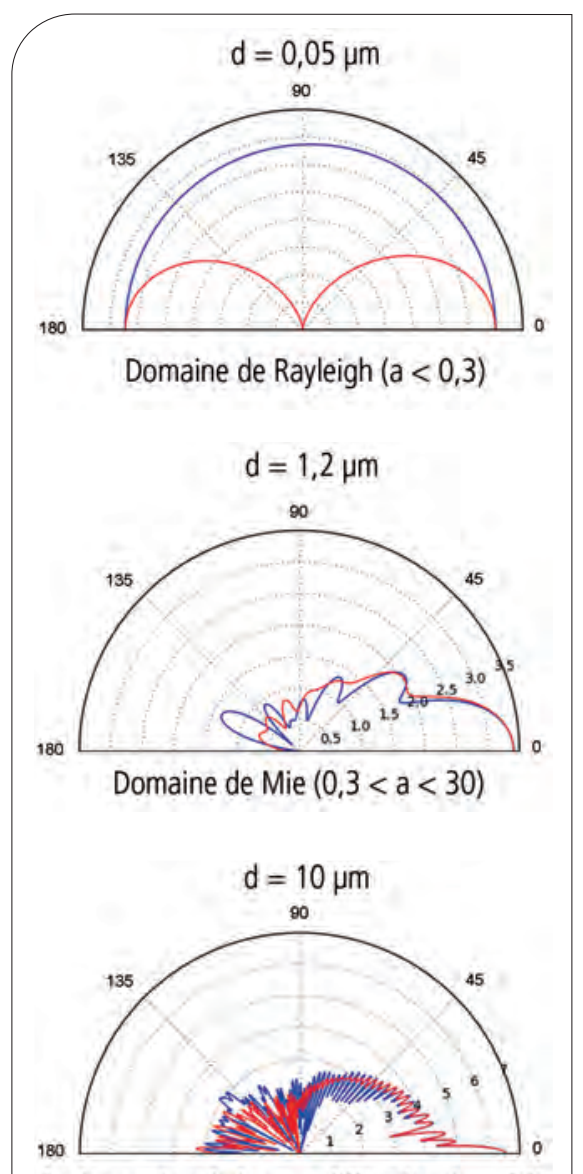

Optique géométrique + diffraction $(a>30)$

Figure 4. Diagrammes de diffusion de gouttelettes d'eau : la courbe bleue désigne l'intensité de la lumière diffusée dont la polarisation est perpendiculaire au plan de diffusion; la courbe rouge est celle pour la polarisation parallèle au plan de diffusion.

quement). Dans cette section, nous décrivons la mise en place d'une technique d'imagerie tomographique qui exploite le principe du taux de polarisation pour déterminer la taille de particules microniques et celui de la corrélation d'images pour déterminer la vitesse des particules (PIV).

La théorie de Mie [Bohren \& Huffman] montre que lorsqu'une particule sphérique reçoit une onde plane incidente, elle diffuse la lumière dans toutes les directions selon une distribution angulaire qui dépend de sa taille. La figure 4 montre un exemple dans le cas de gouttelettes d'eau. On distingue trois domaines de diffusion en fonction du paramètre de taille a de la particule défini par l'équation : $\quad a=\frac{\pi d}{\lambda}$
Les lobes de diffusion observés sur les particules les plus grosses sont directement reliés aux franges d'interférences observées avec la technique de tomographie défocalisée présentée dans la section précédente. Toutefois, dans ce dernier cas, les tailles des particules sont telles qu'une analyse en optique géométrique est suffisante pour interpréter les phénomènes.

Bien sûr, l'intensité de la lumière diffusée est d'autant plus grande que la particule est grosse, mais surtout, la théorie de Mie montre que le diagramme de diffusion est sensible à la polarisation de la lumière de sorte que le taux de polarisation, rapport entre les deux composantes de polarisation, peut être utilisé comme indicateur supplémentaire pour déterminer la taille des particules comme illustré sur la figure 5.

Pour les particules de taille très faible (diffusion moléculaire de Rayleigh, voir dans ce même numéro l'article de Philippe Hervé), la lumière collectée est très fortement polarisée. Le taux de polarisation est alors une fonction strictement monotone du diamètre. Lorsque ce dernier augmente, on observe des oscillations qui introduisent une ambiguïté qui peut être levée en considérant simultanément I'intensité absolue de la particule.

Le dispositif expérimental utilisé consiste en une lentille collectrice qui réalise l'image de la zone de mesure à travers un cube séparateur de polarisation et un ensemble de miroirs qui ramènent les deux images sur le capteur CCD d'une caméra (capteur de taille $24 \times 36 \mathrm{~mm}$ et de 11 MPixels avec une dynamique de 12 bits) en égalant les deux chemins optiques pour être au point sur les voies. Les deux sous-images de polarisation sont alors appariées pixel à pixel par une technique de corrélation avant de déduire le taux de polarisation et l'intensité diffusée. En utilisant un laser Yag impulsionnel, on réalise deux observations successives des particules dans l'écoulement pour déterminer le champ de déplacement (entre deux impulsions cette fois-ci) en appliquant une seconde corrélation ; le champ de vitesse est obtenu par simple division par le $\gg$

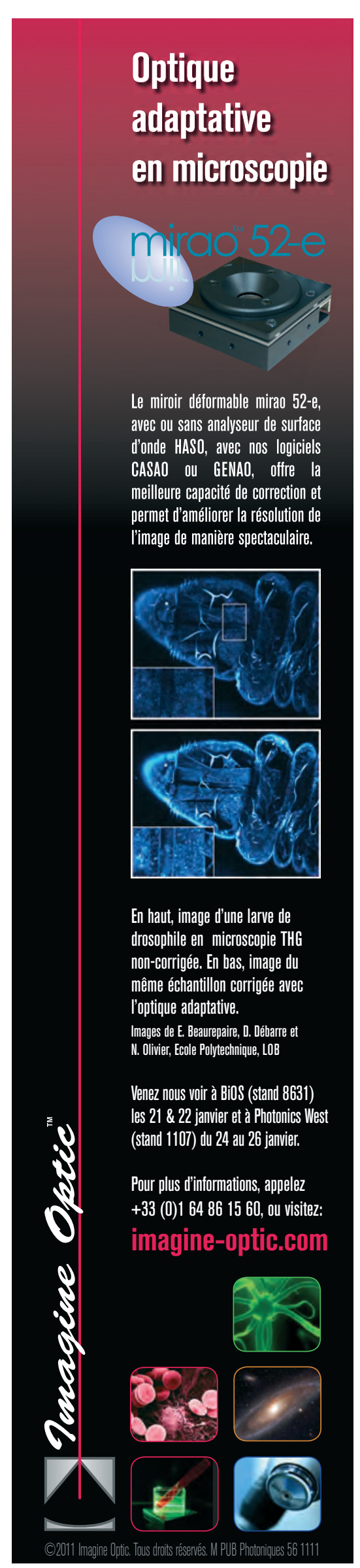



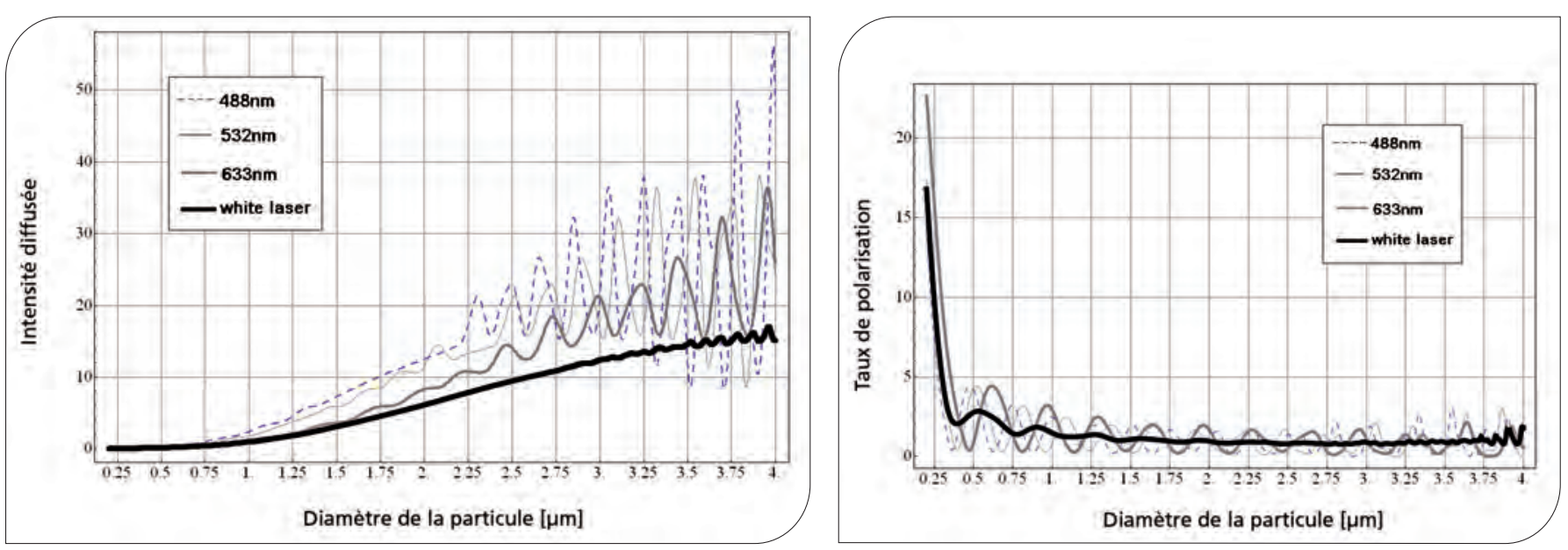

Figure 5. Diffusion à un angle $\theta=80^{\circ}$ à différentes longueurs $d^{\prime}$ ondes.

temps écoulé entre les deux impulsions successives (PIV).

Cette méthode a été appliquée pour étudier la répartition d'un ensemble de gouttelettes d'huile dans un écoulement hélicoïdal généré à l'aval d'une hélice (figure 6). Dans le mouvement de swirl à la sortie de l'hélice, les particules d'huile gagnent une vitesse radiale en fonction de leur masse (diamètre) et de la vitesse tangentielle, à cause de la force centrifuge. Les particules ayant une vitesse radiale suffisante sont alors dirigées vers

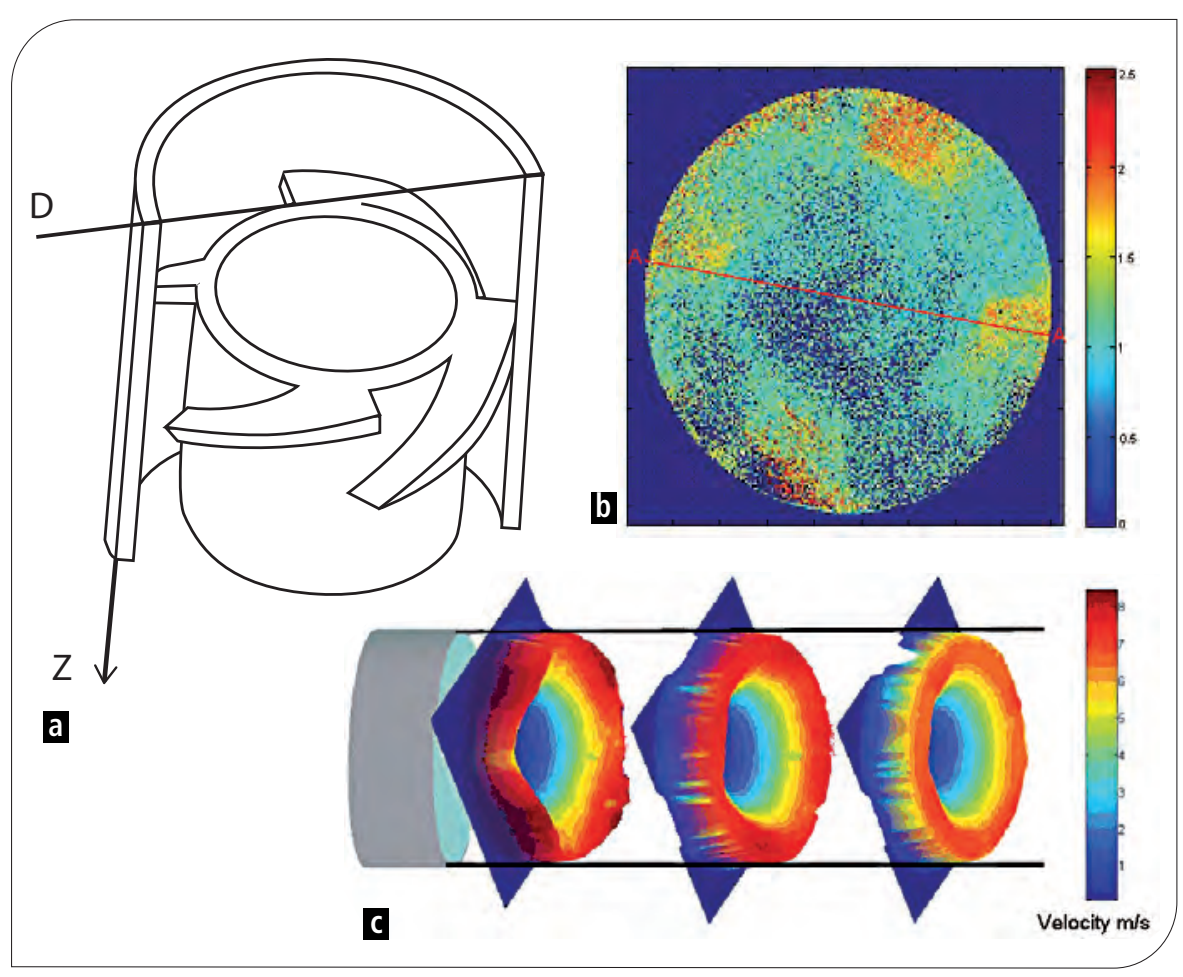

Figure 6. Application de la granulométrie tomographique par taux de polarisation à des gouttelettes d'huile entraînées dans un écoulement hélicoïdal : (a) géométrie de l'hélice ; (b) champ de taille de particules; (c) vitesse des particules dans trois sections en aval de l'hélice. rable à celui du bruit de lecture de la caméra. Les auteurs sont néanmoins parvenus à appliquer cette technique sur des particules de suie de 0,1 $\mu \mathrm{m}$ avec une caméra 16 bits. D'autre part, le processus $d$ 'inversion des données pour remonter à la taille des particules est relativement complexe et demande de grosses capacités de stockage et de calcul pour prendre en compte l'influence de l'angle d'observation, et ceci d'autant plus que la taille des particules augmente. Une simplification pourra peut-être apparaître en utilisant un éclairage non monochromatique tel que celui fourni par un laser blanc; dans ce cas, la figure 5 montre qu'il est possible de réaliser une intégration «naturelle» du paramètre de taille pour un diamètre de particule fixe, et de réduire sensiblement les oscillations du taux de polarisation. Cette piste est actuellement en cours d'évaluation dans nos laboratoires.

\section{Références}

Bohren CF, Huffman DR. Absorption and scattering of light by small particles. John Wiley \& Sons Inc., 1983.

König G., Anders K., Frohn A. A new light-scattering technique to measure the diameter of periodically generated moving droplets. Journal ofAerosol Science. 1986; 17: 157-167.

Onofri F.État de l'art de la granulométrie laser en mécanique des fluides. $9^{e}$ Congrès francophone de vélocimétrie laser. Bruxelles, Belgique, 2004.

Prenel J.P., BaillyY. Recent evolutions of imagery in fluid mechanics: From standard tomographic visualization to $3 \mathrm{D}$ volumic velocimetry. Optics and Lasers in Engineering. 2006; 44: 321-334. 